\title{
CORRESPONDENCE
}

\section{The role of frailty in COVID-19 patients}

\author{
Giuseppe Bellelli ${ }^{1} 2^{*}$ (D) Paola Rebora ${ }^{3}$ and Giuseppe Citerio ${ }^{1}$
}

C 2020 Springer-Verlag GmbH Germany, part of Springer Nature

Dear Editor,

We thank Kow et al. [1] for their interest in our manuscript [2]. In their article, the authors suggest considering frailty and comorbidity as distinct entities and not counting comorbidity into the Frailty Index (FI) construction. This position has some arguments in favor, but also against. The theoretic construct of comorbidity was developed to generate a monodimensional risk profile, assuming that diseases are the most relevant, if not the only, problem of an elder health status [3]. The concept of frailty was instead developed assuming that health status is a multidimensional condition in which comorbidity is one of the problems, but not the unique. Historically, frailty has been evaluated with two different approaches. The frailty phenotype is based on a pre-defined set of criteria that stratifies populations into different risk profiles (i.e., robust, pre-frail and frail) [4]. According to this model, frailty and comorbidity are two independent, mutually interacting conditions. The Rockwood's model [5] lies on the principle that frailty develops as the result of a progressive accumulation of deficits in multiple domains. According to this model, comorbidity is one of the possible dimensions of frailty since it concurs to frailty development by eroding the individuals' biological reserve [3]. The superiority of one model over the other and of the dozen of instruments which have been created to assess frailty has never been demonstrated [3]. Yet, the use of frailty phenotype, as well as of some tools, to assess hospitalized patients during a pandemic, may result challenging. Indeed, the use of specific devices (such as handgrip strength) and the evaluation of tests requiring pencil and paper are not always doable in stressful work environments. Other tools may suffer from a ceiling

*Correspondence: giuseppe.bellelli@unimib.it

1 School of Medicine and Surgery, Acute Geriatric Unit, University of Milano-Bicocca, San Gerardo Hospital, Monza, Italy

Full author information is available at the end of the article effect by classifying all as frails. On the contrary, the use of an electronic FI seems to be more feasible: variables are automatically extracted from medical records and no additional tests are required.

Triggered by Kow's article, we re-analysed our database, building a 19-item FI, that included all variables of our original article [2], except diseases. In a multivariable regression, adjusted for age, sex and comorbidity, the 19-item FI still was independently associated with the outcome (in-hospital mortality or transfer to Intensive Care Unit) (odds ratio, OR 2.29, 95\% confidence intervals, CI 1.39-3.77), while not comorbidity. This suggests that FI may be more predictive than comorbidity. We also assessed the effect of the Clinical Frailty Scale (CFS) in a similar multivariable regression, after removing FI from the list of variables. Here, CFS was independently associated with the outcome (OR 1.09, 95\% CI 1.02-1.16), but lost its statistical significance when adjusting for comorbidity (OR 1.06, 95\% CI 0.99-1.13).

We believe that the dispute regarding instruments has led to a loss of focus on the most important things we can do for older patients in hospital, i.e., to assess frailty. It does not matter what tools we are going to use. What counts is to measure frailty.

\section{Author details \\ ${ }^{1}$ School of Medicine and Surgery, Acute Geriatric Unit, University of Milano- Bicocca, San Gerardo Hospital, Monza, Italy. ${ }^{2}$ Acute Geriatric Unit, San Gerardo Hospital, Monza, Italy. ${ }^{3}$ Bicocca Center of Bioinformatics, Biostatistics and Bioimaging, School of Medicine and Surgery, University of Milano-Bicocca, Monza, Italy.}

\section{Funding}

None.

Compliance with ethical standards

Conflicts of interest

The authors declared that they have no conflict of interest. 


\section{Publisher's Note}

Springer Nature remains neutral with regard to jurisdictional claims in published maps and institutional affiliations.

\section{Accepted: 28 July 2020}

Published online: 1 August 2020

\section{References}

1. Kow CS, Hasan SS (2020) Role of frailty in COVID-19 patients. Intensive Care Med. https://doi.org/10.1007/s00134-020-06172-6
2. Bellelli G, Rebora P, Valsecchi MG, Bonfanti P, Citerio G (2020) COVID-19 Monza Team members. Frailty index predicts poor outcome in COVID-19 patients. Intensive Care Med. 46(8):1634-1636. https://doi.org/10.1007/ s00134-020-06087-2

3. Cesari M, Pérez-Zepeda MU, Marzetti E (2017) Frailty and multimorbidity: different ways of thinking about geriatrics. J Am Med Dir Assoc 18(4):361-364

4. Fried LP, Tangen $C M$, Walston J et al (2001) Frailty in older adults: evidence for a phenotype. J Gerontol A Biol Sci Med Sci 56(3):M146-156

5. Mitnitski A, Song X, Skoog I et al (2005) Relative fitness and frailty of elderly men and women in developed countries and their relationship with mortality. J Am Geriatr Soc 53(12):2184-2189 